\title{
Outer Hair Cell of the Organ of the Corti
}

National Cancer Institute

\section{Source}

National Cancer Institute. Outer Hair Cell of the Organ of the Corti. NCI Thesaurus. Code C33233.

A cell situated on one of three of the most outer layers of the basilar membrane of the cochlea. Each cell has multiple, sensitive strands called stereocilia. In the resting state the stereocilia are leaning on each other in a conical bundle and are embedded in the tectorial membrane. When the cochlea moves in response to sound, a slight shearing force occurs between the basilar and tectorial membranes, the stereocilia bend and send electrical impulses to the brain via the eighth cranial nerve. 